\title{
Nanoflare heating of coronal loops: hydrodynamic response and observational consequences
}

\author{
Y. Taroyan ${ }^{1}$, S. J. Bradshaw ${ }^{2}$, and J. G. Doyle ${ }^{1}$ \\ 1 Armagh Observatory, College Hill, Armagh BT61 9DG, N. Ireland \\ e-mail: yat@arm.ac.uk \\ 2 Space and Atmospheric Physics Group, Blackett Laboratory, Imperial College London, Prince Consort Road, London SW7 2BZ, UK
}

Received 27 June 2005 / Accepted 15 September 2005

\begin{abstract}
The plasma response inside a semicircular coronal loop heated by nanoflares is examined. The loop is thermally and gravitationally stratified. The losses due to thermal conduction and radiation are balanced by localised energy pulses randomly deposited along the loop. The initial stage of the loop evolution during which the temperature along the loop gradually increases from chromospheric to coronal values is completed about 20 minutes after the start of the heating. The random heating produces a thin transition region which is in a continuous motion. The profiles of the C IV, O VI, Ne VIII and Mg X resonance lines, which have peak formation temperatures covering the upper transition region and lower corona, are synthesised. The line shifts and the average shifts are calculated and compared with the observations. It is shown that the nanoflare heating mechanism may reproduce the observed dynamics of the transition region lines. The calculated and previously measured average Doppler shift values are in good agreement for the studied spectral lines except for the C IV line. Possible reasons for this discrepancy are discussed. The study also shows that the nanoflares could contribute to the oscillation power often seen in the $\mathrm{mHz}$ ranges. The power peak of the resulting oscillations is near $2 \mathrm{mHz}$.
\end{abstract}

Key words. Sun: transition region - Sun: corona

\section{Introduction}

Solar observations indicate that the heating of the corona is associated with magnetic activity. The loop-like magnetic structures are identified as the building-blocks of the solar atmosphere (Rosner et al. 1978). The heating mechanism of these building blocks, however, is not yet established. Parker (1988) suggested that the corona is heated by many small and localised bursts of energy called nanoflares. The loops are wound and wrapped around each other due to the continuous shuffling of their photospheric footpoints. This leads to magnetic reconnection between neighbouring loops. According to Parker (1988) the energy released by each event is about $10^{24} \mathrm{erg}$, hence the term nanoflare. Observations of solar flares indicate a power law for their energy distribution with a slope of about $\delta=-1.6$. However, Hudson (1991) pointed out that for the corona to be heated by nanoflares, the slope would need to be more negative than -2 , i.e., the nanoflares must satisfy a different distribution function and, therefore, they may be generated by a different physical mechanism. Studies of small-scale brightenings in the quiet Sun and active regions have shown a power law both steeper and shallower than -2 (Aschwanden \& Parnell 2002). Therefore, no definitive conclusion has been reached at this time. Since the nanoflare concept was put forward, there have been significant developments in both reconnection theory and solar observations (SoHO, TRACE). In the light of these developments, the interaction between theory and observations becomes increasingly important. There are two possible ways in which this interaction can take place.

In the first instance, the observations are used as an input for theoretical modelling to determine the structure and hydrodynamic properties of the plasma occupying the loops. Walsh et al. $(1995,1997)$ have investigated the effects of time dependent heating assuming that the plasma evolves isobarically. The time dependence is either periodic or random whereas the spatial dependence is ignored. They found that the small scale and frequent events are able to maintain a coronal loop at typical coronal temperatures. The plasma may cool to chromospheric temperatures in the case of larger energy events which occur less frequently. Mendoza-Briceño et al. (2002) have studied the effects of spatially and temporally localised heating events. The initial loop atmosphere with an apex temperature of $0.55 \mathrm{MK}$ is maintained by constant uniform heating. The energy pulses are periodic or random, located near the loop footpoints. It is found that these successive localised energy inputs can maintain the plasma along the loop at coronal temperatures. Mendoza-Briceño et al. (2005) extended their study to show that the footpoint heating of coronal loops may also lead to their catastrophic cooling. Muller et al. (2004) showed 
that catastrophic cooling may take place in the case of constant footpoint heating.

In the second case, theoretical ideas are tested by generating observable quantities from models of coronal heating and comparing them with real observations. This approach has been adopted by several authors in different contexts. Cargill (1993, 1994), Cargill \& Klimchuk (1997), Klimchuk \& Cargill (2001) developed a series of models which calculated observables from the nanoflare model for coronal heating. In particular, they calculated the differential emission measure which is an important diagnostic of the coronal heating process and gives indications of the distribution of coronal plasma as a function of temperature and density. In addition, filling factors defined for broadband and narrow-band instruments were calculated. Cargill \& Klimchuk (2004) addressed the impact of power-law heating functions on the emission-measure distribution, density and filling factor. Explosive events in the solar atmosphere were modelled by Sarro et al. (1999) and Innes $\&$ Tóth (1999). Sarro et al. (1999) took into account the effects of non-equilibrium ionisation. It was shown that these events lead to enhancements in the intensities of lines formed between $20000 \mathrm{~K}$ and $200000 \mathrm{~K}$, accompanied by strong nongaussian profiles. Roussev et al. (2001a) extended these simulations into a 2D environment. Analysis of spectral line profiles show that optically thin transition region lines display a net red-shift (Doschek et al. 1976; Dere et al. 1984; Athay \& Dere 1989; Chae et al. 1998; Teriaca et al. 1999a; Peter \& Judge 1999; Doyle et al. 2002). These red-shifts are often accompanied by blue-shifts at higher temperature lines. The redshifts have been observed in coronal holes, quiet sun regions, and active regions. There have been many attempts to understand their physical origin. Pneuman \& Kopp (1977) and later Athay \& Holzer (1982) hypothesised that the red-shifts were caused by the return of spicular material. A numerical study of a spicule upflow followed by a cooling phase performed by Mariska (1987) could not reproduce the observed behaviour. McClymont \& Craig (1987) showed that asymmetric heating of coronal loops may drive siphon flows through coronal loops. As the condensing leg with the downflow dominates the emission, a net red-shift would be observed. However, the observations seem inconsistent with a steady flow model. Mariska (1988) found that time-dependent asymmetric heating causes red-shifts and blue-shifts. However, this model predicts blueshifts for middle transition region lines, contrary to the observations. Spadaro et al. (1990) showed that the inclusion of nonequilibrium ionisation has little effect on the results. Hansteen (1993) interpreted the red-shifts as a manifestation of downward propagating acoustic waves. The waves are assumed to be generated near the loop apex as a result of nanoflares or some other form of episodic heating which is superimposed on the uniform constant background heating. After the initial stage of evolution is completed, the middle transition region lines are predominantly blue-shifted, which is again inconsistent with the observations. Teriaca et al. (1999b), Spadaro et al. (2003) studied the consequences of a single heating pulse in a 1D loop. The initial stages of evolution are followed by quasisteady flows when the heating pulse is completed. Meanwhile, according to observations, the flows in the transition region lines are highly dynamic and variable. Recently Gudiksen \& Nordlund (2005) modelled a small part of the corona in a 3D computational box using a potential extrapolation from a SoHO/MDI magnetogram. The heating of a small active region by the field line braiding mechanism was simulated. A realisticlooking corona consisting of a number of loop-like structures was generated. Peter et al. (2004) extended this study to synthesise emission line spectra. They found a clear red-shift for almost all emission lines from the low corona and transition region (EUV; 500-1500 ̊). The results of this numerical experiment look promising despite the limited vertical resolution of the transition region $(\geq 150 \mathrm{~km})$.

In the present work we apply the nanoflare heating mechanism to a single 1D loop model. The nanoflares are represented as episodic heating events randomly distributed along the loop. The evolution of the loop from an initial chromospheric temperature of $20000 \mathrm{~K}$ is studied. The hydrodynamic implications of this mechanism are examined. The model contains an adaptive grid with a very high spatial resolution in the narrow transition region. This allows us to study the dynamic behaviour of the transition region and adequately synthesise the emission line profiles. The observational consequences of the nanoflare model in terms of the line profiles and Doppler shifts are discussed. The time series of the line shifts are Fourier analysed to see if the nanoflares could be responsible for some of the oscillations observed in the transition region and corona.

\section{Loop modelling and the initial state}

In this section we introduce the loop model and the governing equations of the plasma motion inside the loop. It is assumed that the motion inside the loop is essentially guided along the magnetic field lines. Therefore, the loop can be represented as a 1D semicircular magnetic flux tube with footpoints anchored in the deep chromosphere. In addition, a constant cross-sectional area of the loop and no inclination at the footpoints are assumed. The plasma motion inside the loop is governed by the 1D hydrodynamic equations for the conservation of mass, momentum and energy:

$$
\begin{aligned}
& \frac{\partial \rho}{\partial t}+\frac{\partial \rho v}{\partial s}=0 \\
& \frac{\partial \rho v}{\partial t}+\frac{\partial \rho v^{2}}{\partial s}=-\frac{\partial p}{\partial s}+\rho g_{\|} \\
& \frac{\partial e}{\partial t}+\frac{\partial}{\partial s}[(e+p) v]=\rho v g_{\|}+\mathcal{S} \\
& e=\frac{p}{\gamma-1}+\frac{\rho v^{2}}{2}
\end{aligned}
$$

In the above Eqs. (1)-(4) $t$ is time, $s$ is the distance along the loop, and $\rho, v, e, p$ are the density, velocity along the loop, energy density and pressure, respectively. The quantity $\mathcal{S}$ represents the net effect of sources and sinks of energy, $\gamma$ is the adiabatic index,

$g_{\|}=-g_{\odot} \cos \left(\frac{\pi s}{L}\right)$ 

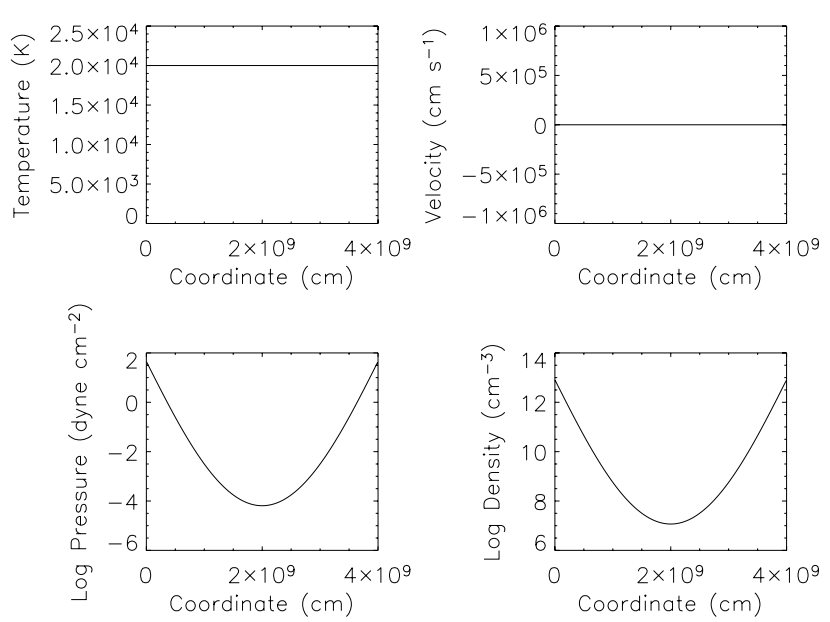

Fig. 1. A $40 \mathrm{Mm}$ long loop with an initial temperature of $T=2 \times 10^{4} \mathrm{~K}$ is in hydrostatic equilibrium.

is the gravitational acceleration along the loop and $g_{\odot}=2.74 \times$ $10^{4} \mathrm{~cm} \mathrm{~s}^{-2}$. Here $L$ is the total length of the loop. The set of Eqs. (1)-(4) is completed by the perfect gas law

$p=\frac{R}{\tilde{\mu}} \rho T$,

where $T$ is the temperature, $R$, and $\tilde{\mu}$ are the gas constant and the mean molecular weight, respectively.

The term $\mathcal{S}$ in Eq. (3) can be represented in the form

$\mathcal{S}=\mathcal{H}-\frac{\partial F_{\mathrm{c}}}{\partial s}-n^{2} \Lambda$.

The first term in Eq. (7) is the heating rate to be specified. The second term represents the thermal conduction along the magnetic field; $F_{\mathrm{c}}$ is the conductive flux along the field given by

$F_{\mathrm{c}}=-\varkappa T^{5 / 2} \frac{\partial T}{\partial s}$

where $\varkappa=10^{-6} \mathrm{erg} \mathrm{s}^{-1} \mathrm{~K}^{-1} \mathrm{~cm}^{-1}$ is the coefficient of thermal conduction along the field. The third term represents optically thin radiative losses: $n$ is the number density and $\Lambda=\Lambda(T)$ is the radiative loss function. The details of how this function is implemented in our calculations are described in Bradshaw \& Mason (2003).

We consider a $40 \mathrm{Mm}$ long loop. The loop is initially in hydrostatic equilibrium is shown in Fig. 1. The equilibrium is derived by integrating the hydrostatic equations of pressure and energy. It is kept at a constant temperature of $T=2 \times 10^{4} \mathrm{~K}$. The heating $\mathcal{H}$ and the radiative loss function $\Lambda$ are initially switched off and there are no thermal losses because of the constant temperature. This means that the net effect of sources and sinks of energy $\mathcal{S}$ is initially set to zero in Eq. (3).

\section{Nanoflare heating and the hydrodynamic evolution of the loop}

In this section we investigate the hydrodynamic response of the loop to the nanoflare heating. The nanoflares are represented in the form of episodic heating events which occur randomly in time and have a random duration. This type of modelling has already been done in the past (Walsh et al. 1997;
Mendoza-Briceño et al. 2002). An extra constant background heating term was added which would maintain the loop atmosphere at coronal temperatures in the absence of the nanoflares. In the present paper we assume that the entire heating is due to the nanoflares. Thus the heating rate is represented in the following form:

$\mathcal{H}=\sum_{i=1}^{n} \mathcal{H}_{i}(t, s)$

where $\mathcal{H}_{i}(t, s), i=1, \ldots, n$ are the discrete episodic heating events and $n$ is the total number of the events. The present model therefore allows us to address various important aspects of the hydrodynamic response of the loop to nanoflares such as the evolution of the loop from chromospheric to coronal temperatures, the formation of the transition region and its dynamic behaviour in time.

In recent years there have been a number of speculations regarding the distribution of the heating function along the loops. Priest et al. (1998) have argued that the heating is uniform along the loop, whereas the TRACE observations of isothermal loops favour the idea that the heating is concentrated near the loop footpoints (Aschwanden et al. 2001). The latter view is supported by the numerical simulations of Gudiksen et al. (2005). Recent work by Ugarte-Urra et al. (2005) where they derive the electron density variability along a loop, coupled with one dimensional hydrodynamic modelling also suggest a best fit case where the total energy input is directed preferentially to the loop footpoint with the heating rate being three times larger at the base than at the apex.

It is plausible that different types of loops are heated in different ways. In the present work we assume, for simplicity, that the episodic heating events are distributed randomly along the entire length of the loop above the chromosphere. Each heating event has the form

$\mathcal{H}_{i}(t, s)= \begin{cases}E_{0} \sin \frac{\pi\left(t-t_{i}\right)}{\tau_{i}} \exp \frac{\left|s-s_{i}\right|}{s_{h}}, & t_{i}<t<t_{i}+\tau_{i}, \\ 0, & \text { otherwise }\end{cases}$

where $E_{0}$ is the maximum heat input and $s_{\mathrm{h}}$ is the heating scale length. The offset time $t_{i}$, the duration $\tau_{i}$, and the location $s_{i}$ of each heating event $i$ can be written as

$t_{i}=\alpha_{i} t_{\mathrm{total}}, \quad \tau_{i}=\beta_{i} \tau_{\max }, \quad s_{i}=s_{\min }+\gamma_{i}\left(L-2 s_{\min }\right)$,

where $\alpha_{i} \beta_{i}, \gamma_{i}$ are random numbers between 0 and $1, t_{\text {total }}$ is the total duration of the simulations, $\tau_{\max }$ is the maximum duration of a single heating event and $s_{\min }$ is the minimum height below which the heating is switched off. We assume that the minimum height of the nanoflare heating is located at the top of the chromosphere. The chromosphere is kept at a constant temperature of $T_{\mathrm{ch}}=2 \times 10^{4} \mathrm{~K}$.

Although the solar transition region and corona are clearly in a dynamic state, in the present paper we make the simplifying assumption that the ion populations and radiative losses remain in equilibrium. Recently Bingert et al. (2004) have argued that this assumption could be justified for a number of ions (e.g., C II, C IV, O VI, Ne XIII) when the flow speeds are moderate inside the loop. On the other hand, the results of, e.g., 

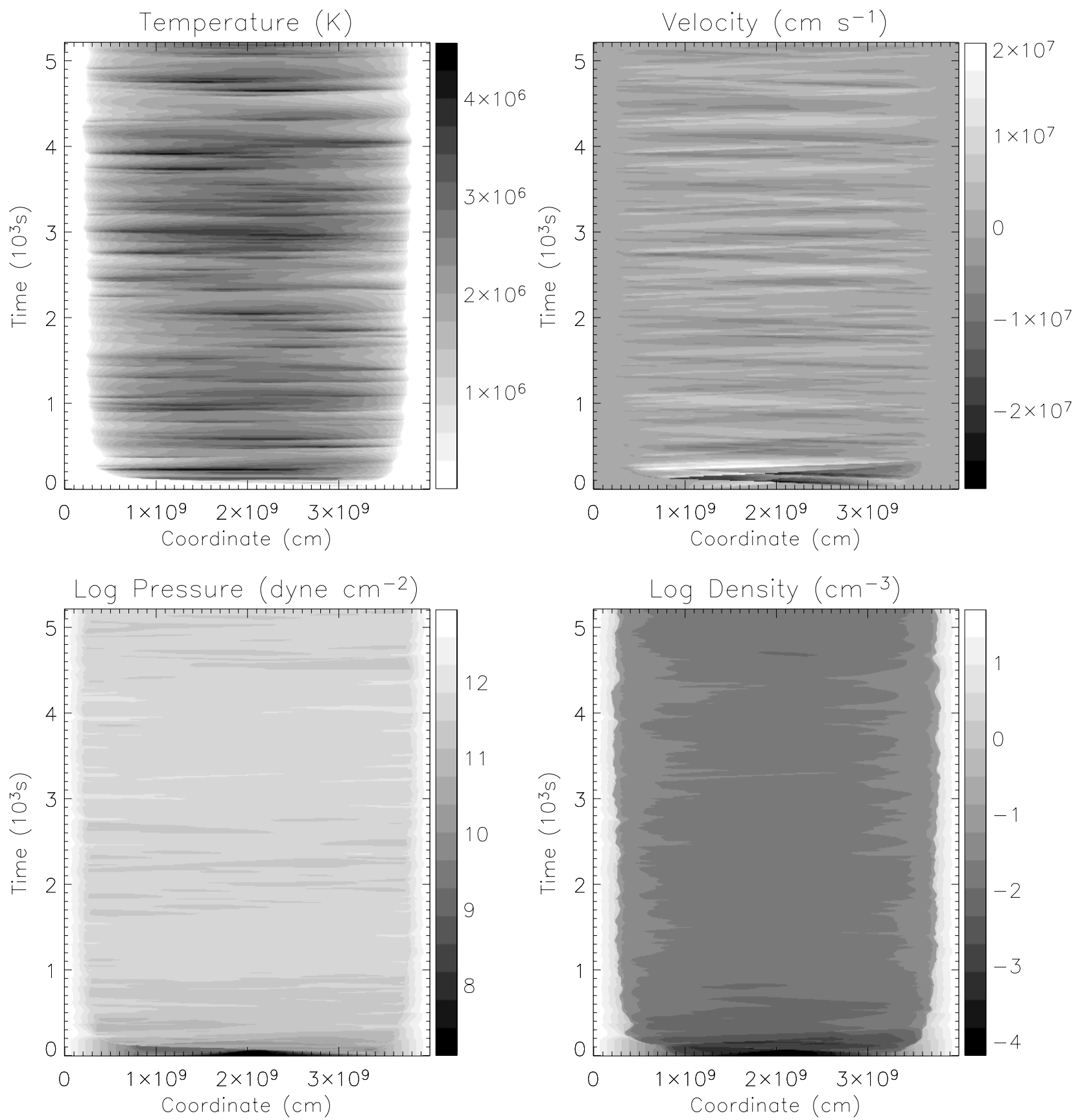

Fig. 2. The evolution of temperature, velocity, pressure and density inside the loop from $t=0 \mathrm{~s}$ to $t=5200 \mathrm{~s}$. The dark colours indicate high temperature, low pressure, low density and negative velocities, whereas the bright colours indicate low temperature, high pressure, high density and positive velocities.

Bradshaw \& Mason (2003a,b), Bradshaw et al. (2004) showed that the transition region and coronal ion populations can depart from equilibrium for sub-sonic flows. We plan to investigate the effects of non-equilibrium ion populations in a forthcoming paper. The radiative loss function $\Lambda=\Lambda(T)$ is suppressed below the chromospheric temperature of $T_{\text {ch }}=2 \times 10^{4} \mathrm{~K}$. This helps to avoid the numerical instability due to the $n^{2}$ dependence of radiation and it reflects the fact that the chromosphere is mostly optically thick and so does not radiate as strongly as the overlying atmospheric layers (see, e.g., Klimchuk et al. 1987; Antiochos et al. 1999; Spadaro et al. 2003; Bradshaw \& Mason 2003a,b).
The following values are set for the parameters in Eqs. (10)

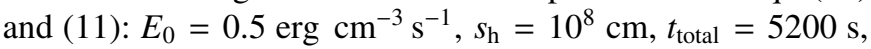
$\tau_{\text {max }}=40 \mathrm{~s}, s_{\text {min }}=2 \times 10^{8} \mathrm{~cm}$. The total number of random heating pulses deposited along the loop between the start at $t=0 \mathrm{~s}$ and the end of the simulations at $t=5200 \mathrm{~s}$ is $n=104$. Equations (1)-(3) are integrated using HYDARD (Bradshaw \& Mason 2003a,b). An adaptive regridding scheme is used in order to ensure that the small-scale spatial features along the loop, which arise due to the random nanoflare heating, are correctly resolved.

The time-distance plots in Fig. 2 show the evolution of the temperature, velocity, pressure and density inside the loop. 
There are two stages in the loop evolution: the initial stage (from 0 to $1200 \mathrm{~s}$ ) characterised by temperature increase and the main stage (from $1200 \mathrm{~s}$ until the end of the simulations). During the initial stage the apex temperature increases and quickly reaches coronal values. In contrast to the apex, the temperature increase near the footpoints is slower. Figure 2 shows that the temperature increase near the footpoints of the loop is completed by $t=1200 \mathrm{~s}(20 \mathrm{~min})$. The nanoflare heating leads to the formation of a narrow transition region which separates the bright regions representing the cool chromosphere from the darker regions representing the corona. The adaptive regridding scheme allows us to accurately trace the position of the transition region as the plasma inside the loop evolves. The typical spatial resolution inside the transition region is on the order of $100 \mathrm{~m}$. Figure 2 shows that the transition region is in a continuous motion. The displacement can be as large as $1 \mathrm{Mm}$. The temperature in the coronal part of the loop is characterised by rapid changes. The apex temperature varies between 2 and $4 \mathrm{MK}$. Thus the nanoflare heating is able to sustain the loop at coronal temperatures by balancing the losses due to optically thin radiation and thermal conduction. It also leads to the formation of a dynamic and narrow transition region.

The initial flows with large velocities (over $100 \mathrm{~km} \mathrm{~s}^{-1}$ ) which can be seen at the beginning of the simulations, gradually settle down during the initial stage of the loop evolution. However, these longitudinal flows remain highly variable until the end of the simulations. The flow speeds are the highest in the coronal part of the loop where they can vary by up to $50 \mathrm{~km} \mathrm{~s}^{-1}$ in less than a minute.

Figure 2 also displays the evolution of pressure and density along the loop. These quantities increase in the coronal part of the loop during the initial heating stage and remain nearly constant at the footpoints.

Additional simulations show that the loop is subject to catastrophic cooling at certain stages of its evolution if the heating is concentrated near the footpoints. This is in agreement with the results of Mendoza-Briceño et al. (2005). It is also possible to sustain the loop at higher (lower) temperatures by increasing (decreasing) the total number of pulses $n$ or the maximum duration of the events $\tau_{\max }$. However, the detailed examination of these problems is beyond the scope of the present paper.

\section{Observational consequences of nanoflare heating}

Figure 2 shows the hydrodynamic evolution of the loop in response to the heating by nanoflares. However, one cannot compare these results with real observations. In order to do that we need to express the results in terms of observable quantities such as emission line profiles and Doppler shifts.

\subsection{Line synthesis}

The following procedure is applied to calculate the line profiles. We select some of the lithium-like ions which have formation temperatures covering the upper transition region and the lower corona. The ion populations are calculated using
HYDRAD. The atomic data for the calculation of emissivities are provided by the recent version of the CHIANTI atomic database (Young et al. 2003). The emissivity of a resonant line per unit interval of wavelength is given by the expression (see, e.g., Withbroe 1981; Spadaro et al. 1990; Sarro et al. 1999)

$E_{\lambda} \propto \frac{h c}{\lambda} \frac{\Omega}{\omega} \frac{N_{1}}{N_{\text {ion }}} \frac{N_{\text {ion }}}{N_{\text {elem }}} \frac{N_{\text {elem }}}{N_{\mathrm{H}}} N_{\mathrm{H}} N_{\mathrm{e}} \frac{\exp \frac{-W}{k_{\mathrm{B}} T}}{\sqrt{T}} \Phi(\lambda)$,

where $h$ is the Planck constant, $c$ is the speed of light, $\Omega$ is the collisional strength, $\omega$ is the statistical weight of the lower level, $N_{1} / N_{\text {ion }}$ is the ratio of ions responsible for the emission in the ground state relative to the total number of ions per unit volume, $N_{\text {ion }} / N_{\text {elem }}$ is the relative abundance of the ionic species, $N_{\text {elem }} / N_{\mathrm{H}}$ is the abundance of the element relative to hydrogen, $N_{\mathrm{H}}$ is the proton density, $N_{\mathrm{e}}$ is the electron density, $W$ is the energy difference between the upper and lower levels, $k_{\mathrm{B}}$ is the Boltzman constant, and

$\Phi(\lambda)=\frac{1}{\Delta \lambda_{0} \sqrt{\pi}} \exp -\left[\frac{\lambda-\lambda_{0}-\lambda_{\mathrm{s}}}{\Delta \lambda_{0}}\right]^{2}$

is the line broadening function. In the above definition $\lambda_{0}$ is the rest wavelength of the resonance line, $\lambda_{\mathrm{s}}=\left(\lambda_{0} / c\right) v_{\mathrm{p}}$ is the Doppler shift corresponding to the bulk velocity of the plasma projected on the line of sight $v_{\mathrm{p}}, \Delta \lambda_{0}$ is the Doppler width of the line:

$\Delta \lambda_{0}=\frac{\lambda_{0}}{c} \sqrt{\frac{2 k_{\mathrm{B}} T}{m_{i}}}$

and $m_{i}$ is the mass of the ion producing the spectral line. The results of the preceding section have shown that the initial stage of the loop evolution is completed by $t=1200 \mathrm{~s}$. In the following analysis the spectral line profiles are calculated for the main stage, i.e., from $t=1200 \mathrm{~s}$ until the end of the simulations at $t=5200 \mathrm{~s}$. The line intensities are calculated in normalised units. The normalisation is with respect to the maximum intensity of the corresponding line emitted at $t=1200 \mathrm{~s}$. The line shifts can be expressed both in wavelength units $(\AA)$ and in velocity units $\left(\mathrm{km} \mathrm{s}^{-1}\right)$. Positive velocities represent red-shift and negative velocities represent blue-shift. The Doppler shift depends on the positioning angles of the loop on the solar disk. For simplicity, only loops at the disk centre are considered. The total intensity of a spectral line integrated along a loop segment $\left[s_{\text {left }}, s_{\text {right }}\right]$ is

$I(\lambda, t)=\int_{s_{\text {left }}}^{s_{\text {right }}} E(\lambda, t, s) \mathrm{d} s$.

Previous works dealing with the line synthesis have mainly considered the intensity integrated along the entire loop from 0 to $L$ (Spadaro et al. 1990; Sarro et al. 1999; Teriaca et al. 1999b). In the present work, the intensities near the footpoints of the loop are studied. For the first segment near the left footpoint $s_{\text {left }}=2 \mathrm{Mm}, s_{\text {right }}=5 \mathrm{Mm}$. For the second segment near the right footpoint $s_{\text {left }}=35 \mathrm{Mm}, s_{\text {right }}=38 \mathrm{Mm}$. These two segments surround the transition region where the selected resonance lines have their emission peak (about 1-2 orders of magnitude higher than the emission in the rest of the loop). 
Also, the Doppler shift near the footpoints is expected to be relatively large due to the alignment with the line of sight. The projection of these segments on the solar disc is about $800 \mathrm{~km}$ which is within the resolution limit of current spectrometers.

For the range of electron densities considered here, the assumptions within CHIANTI are valid, however, as Doyle et al. $(2005 a, b)$ has shown considering the electron density dependence of dielectronic recombination coupled with collisional ionisation from metastable levels can change the line intensity of many resonance lines at higher electron densities.

\subsection{Dynamics of the transition region lines}

The selected lines are CIV 1548.20 ̊, O VI $1031.93 \AA$, Ne VIII $770.43 \AA$ and Mg X $624.97 \AA$. These lines represent the lithium isoelectronic sequence and have peak formation temperature of $\log T_{\mathrm{CIV}}=5, \log T_{\mathrm{OVI}}=5.5, \log T_{\mathrm{Ne} \mathrm{VIII}}=5.9$, $\log T_{\mathrm{Mgx}}=6.1$. Therefore, they can be used to study the dynamics of the upper transition region and lower corona. Figure 3 displays the selected line profiles. These plots show that the C IV, O VI, Ne VIII and Mg X line emissions coming out from the loop due to the nanoflare heating do not vary too much during the main stage of the loop evolution. The Gaussian profiles seen in Fig. 3 also suggest that the line shifts have single components. This is due to the small scale of the energies involved. Higher energy events such as explosive events are characterised by highly non-Gaussian line profiles. Sarro et al. (1999) and Roussev et al. (2001b) found that soon after the onset of the high energy injection the line profile experiences a sudden increase in intensity and splits into two components with opposite wavelength shifts. The resulting loop emission can outshine by more than two orders of magnitude the emission from the quiet loop. In the present case there is no splitting of the line profiles. Figure 4 shows the line shift in C IV, $\mathrm{O}$ VI, Ne VIII and Mg X plotted as a function of time. The plots in Fig. 4 show that in all cases the line shift is highly variable. It typically varies between -10 and $20 \mathrm{~km} \mathrm{~s}^{-1}$ and it can change by $20 \mathrm{~km} \mathrm{~s}^{-1}$ in less than a minute. These results are consistent with the observations of Doyle et al. (2002) who studied the temporal variability in the Doppler shift of transition region lines. High cadence datasets in C III, O VI and Ne VIII were analysed in an effort to establish the extent of the Doppler shift variability in different regions of the quiet Sun. Within each of these regions short time-scale variability for the Doppler shift was established. In some cases the line shift could change by $15 \mathrm{~km} \mathrm{~s}^{-1}$ in less than a minute. It was found that the line shift in $\mathrm{C}$ III (which has a peak formation temperature of $\log T_{\mathrm{C} \text { III }}=4.8$ ) ranges from $-2 \mathrm{~km} \mathrm{~s}^{-1}$ to $20 \mathrm{~km} \mathrm{~s}^{-1}$ and the line shift in O VI could range from $-2 \mathrm{~km} \mathrm{~s}^{-1}$ to $15 \mathrm{~km} \mathrm{~s}^{-1}$. In the case of Ne VIII substantial difference was found between internetwork and bright network regions with the bright network being more red-shifted. The plots displayed in Fig. 4 compare well with the corresponding observational plots for the line shifts displayed in their Figs. 1-3. These results show that the nanoflare model is able to reproduce the dynamics of the transition region lines observed by Doyle et al. (2002).

\subsection{Average Doppler shifts}

Another important question concerns the average Doppler shifts. Much effort has been devoted to the acquisition and understanding of the average Doppler shifts of emission lines formed in the solar corona and transition region (Doschek et al. 1976; Mariska et al. 1978; Feldman et al. 1982; Dere et al. 1984; Klimchuk 1987; Athay \& Dere 1989; Rottman et al. 1990; Brekke et al. 1997; Chae et al. 1998; Teriaca et al. 1999a; Peter \& Judge 1999; Doyle et al. 2002). These observations have revealed that the transition region lines in the chromospheric network are predominantly red-shifted. This observational fact has long been recognised as an important clue to the processes in the solar atmosphere, prompting several authors to build physical models of a remarkably wide variety to try to account for this phenomenon. Some of the ideas put forward in the past are summarised in the Introduction.

The present nanoflare heating model reproduces the redshifts in the transition region lines seen in the observations. The results of some of the above mentioned observational studies are compared with the results of the present paper in Table 1. For C III, Doyle et al. (2002) found a range of average redshifts: $8 \mathrm{~km} \mathrm{~s}^{-1}$ in a network regions, $2 \mathrm{~km} \mathrm{~s}^{-1}$ in an internetwork region, and $13 \mathrm{~km} \mathrm{~s}^{-1}$ at the boundary of a bright network region. Comparing the C IV modelling results with the corresponding observational results, we see that the average Doppler shift predicted by the model is somewhat lower than the values reported in the literature. This discrepancy could be attributed to the fact that the present model does not take into account the non-equilibrium ionisation. Another contributing factor could be the form of the heating function. The present model assumes that the heating is randomly distributed along the entire length of the loop above the minimum height. A heating function concentrated near the footpoints of the loop could increase the average red-shift in the C IV emission line. These effects need to be taken into account in future studies. The agreement is better in the case of the O VI line. For Ne VIII and Mg X the observations show a range of values. Doyle et al. (2002) have found an average red-shift in a network region and blue-shift in a bright network region.

Most observations indicate that the average Doppler shifts decrease or become negative as we move from the lines formed at transition region temperatures to the lines formed at higher coronal temperatures. The present model reproduces this trend seen in the observations. The average Doppler shift values for Ne VIII and Mg X could become negative if the heating takes place at the footpoints of the loop: the pressure increase at the footpoints due to the heating could drive flows in opposite directions which would contribute to the increase of the red-shift at lower temperatures (C IV and O VI lines) and the increase of the blue-shift at higher temperatures ( $\mathrm{Ne} \mathrm{VIII} \mathrm{and} \mathrm{Mg} \mathrm{X}$ lines).

\subsection{Oscillation signatures}

Finally, we examine the possibility of the nanoflare heating mechanism being related to the oscillations seen in the solar transition region and corona. 

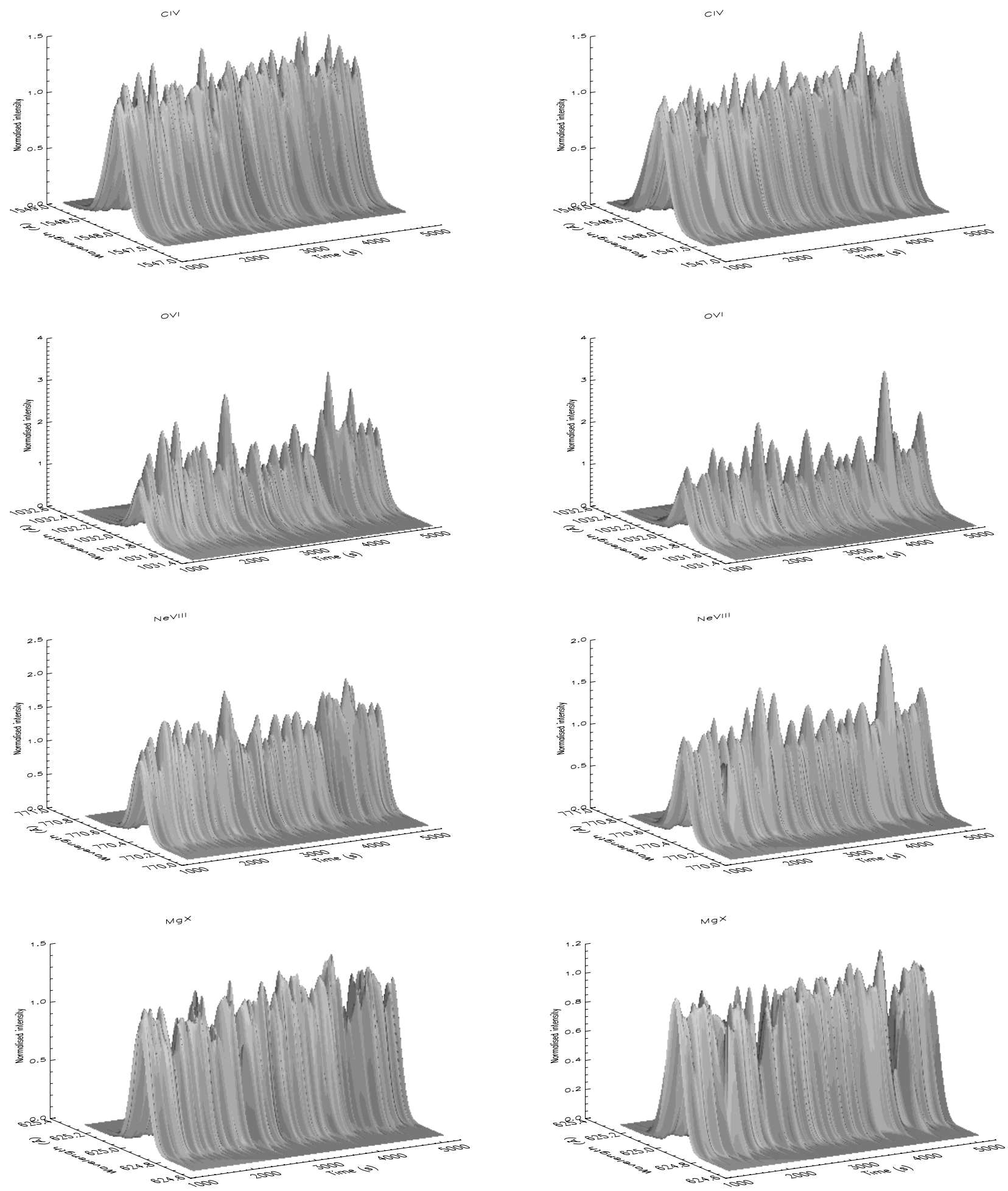

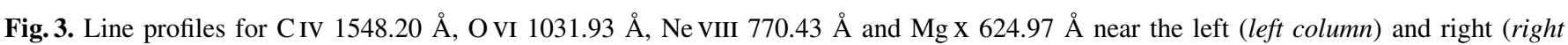
column) footpoints. The calculations are done from $t=1200 \mathrm{~s}$ to $t=5200 \mathrm{~s}$. The intensity of each line is normalised with respect to the corresponding maximum intensity at $t=1200 \mathrm{~s}$.

For the C IV resonant line, Athay \& White (1979) found that small-amplitude aperiodic fluctuations characterised most of the data. About 20 percent of the data showed fluctuations with power in the $3-5 \mathrm{mHz}$ range. They interpreted the C IV results as suggesting that sound waves are common at $10^{5} \mathrm{~K}$ in the transition region, but that often their passage through the upper chromosphere and transition region has destroyed their periodicity. Judge et al. (1997) have reported on SUMER 
Y. Taroyan et al.: Nanoflare heating of coronal loops: hydrodynamic response and observational consequences
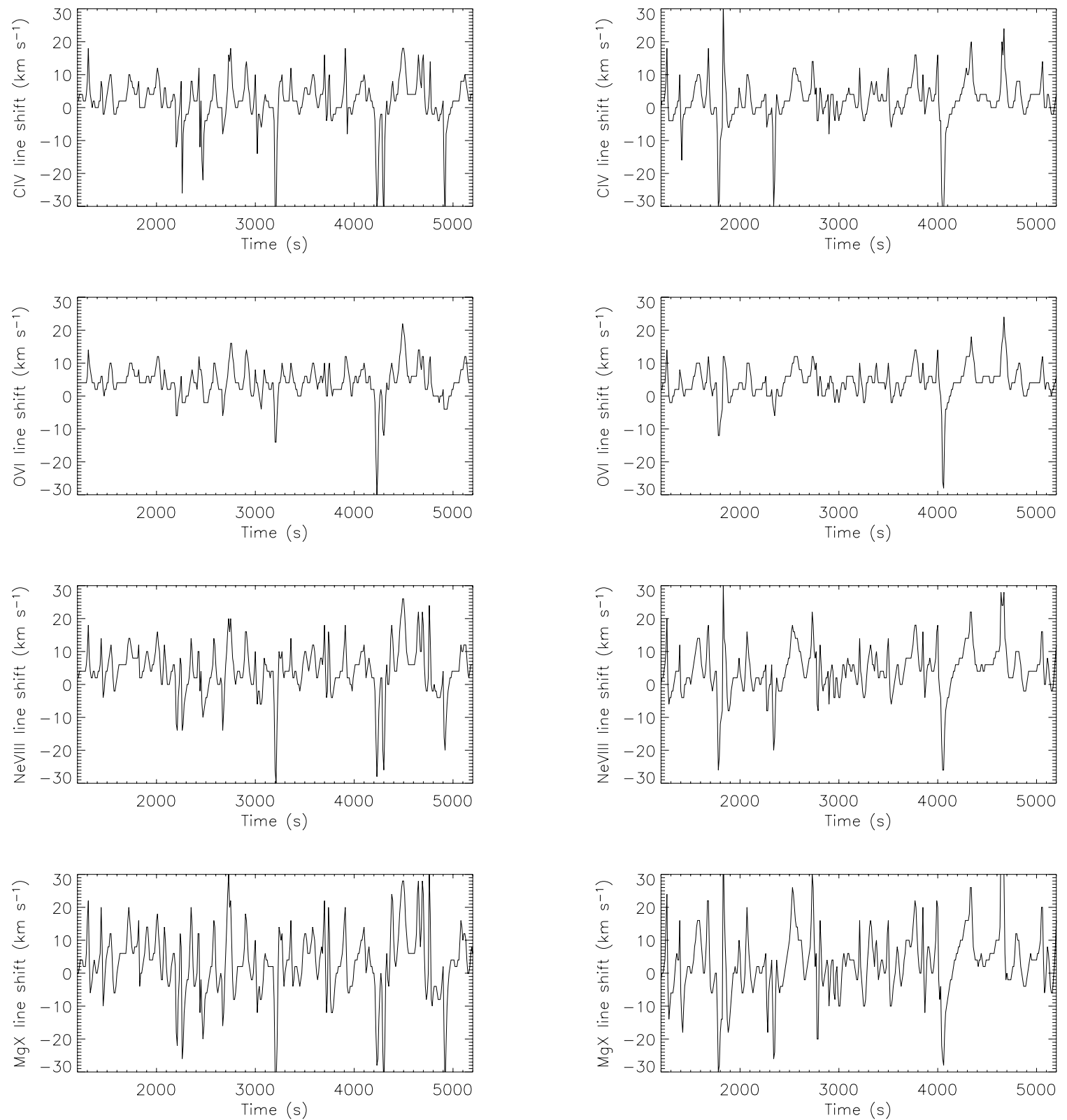

Fig. 4. The Doppler shifts of the C IV, O VI, Ne VIII and Mg X resonance lines near the left (left column) and right (right column) footpoints measured in $\mathrm{km} \mathrm{s}^{-1}$ as functions of time measured in $\mathrm{s}$. The line shifts are plotted from $t=1200 \mathrm{~s}$ to $t=5200 \mathrm{~s}$.

Table 1. Average Doppler shifts in $\mathrm{km} \mathrm{s}^{-1}$. Positive velocities represent red-shifts and negative velocities represent blue-shifts.

\begin{tabular}{lcccc}
\hline \hline Source & C IV & O VI & Ne VIII & Mg X \\
\hline Brekke et al. (1997) & 6 & 5 & 5 & 6 \\
Chae et al. (1998) & 9.6 & 8.7 & 5.3 & 3.8 \\
Teriaca et al. (1999a) $^{a}$ & $10.2 \mathrm{AR}, 4.9 \mathrm{QS}$ & $8.7 \mathrm{QS}$ & $-7.8 \mathrm{AR},-1.9 \mathrm{QS}$ & - \\
Peter \& Judge (1999) $^{\text {Doyle et al. (2002) }}$ & 4.5 & 5.6 & -2.4 & -4.5 \\
Present modelling results & - & $4.8 \mathrm{WNR}, 10 \mathrm{SNR}$ & $-8 \mathrm{NR}, 5 \mathrm{BNR}$ & - \\
Left footpoint & 1.9 & 4.2 & 4.1 & 2.8 \\
Right footpoint & 2.1 & 4.2 & 4.2 & 3 \\
\hline
\end{tabular}

a AR refers to an active region and QS refers to the quiet Sun.

$b$ WNR refers to a weak network region, SNR stands for a strong network region and BNR stands for a bright network region. 

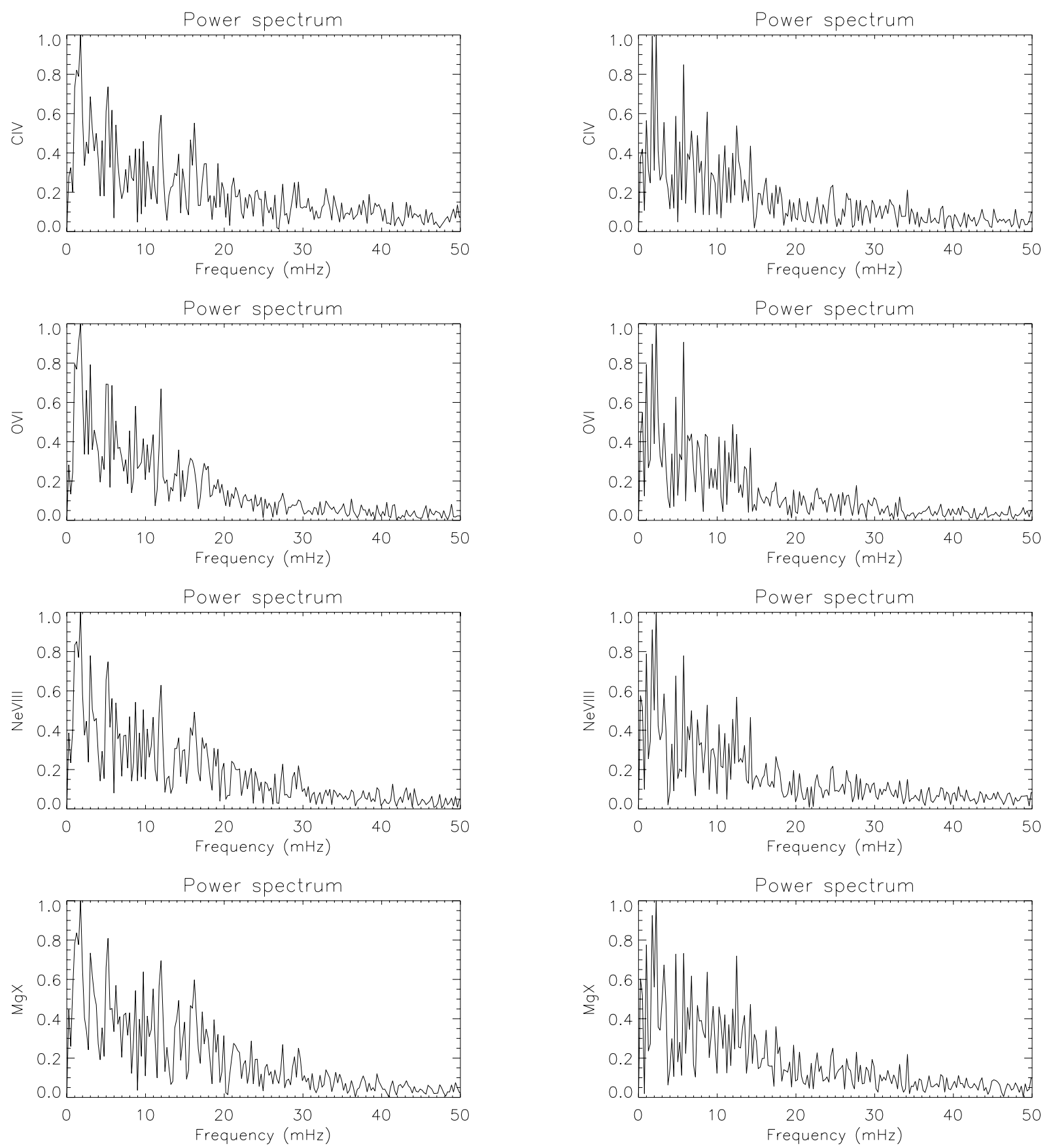

Fig. 5. Normalised power spectra derived for the time series of the Doppler shifts of the CIV, O VI, Ne VIII and Mg X resonance lines near the left (left column) and right (right column) footpoints. The normalisation is with respect to the highest peak in the spectrum. Frequency is measured in $\mathrm{mHz}$.

observations of small-amplitude $\left(2-5 \mathrm{~km} \mathrm{~s}^{-1}\right)$ coherent oscillations of $\sim 130$ s period in Doppler shifts of $\mathrm{Si} \mathrm{III}\left(\log T_{\mathrm{Si} \text { III }}=\right.$ 4.6) between regions of bright network elements. Doyle et al. (1998) analysed the power spectra for line intensities of several lines formed in the transition region around 100000 to $250000 \mathrm{~K}$. Clear periods were observable in the $2-5 \mathrm{mHz}$ range with the largest power peak at $3 \mathrm{mHz}$. A smaller peak was seen at around $5.0 \mathrm{mHz}$. In one dataset a period approaching $10 \mathrm{~min}(1.5 \mathrm{mHz})$ was present. Banerjee et al. (2001) examined the time-series of spectral data obtained from CDS and
SUMER. The transition region lines showed intensity and velocity power in the $2-4 \mathrm{mHz}$ range.

In order to establish the presence of any oscillations in our model, we have Fourier analysed the Doppler shift time-series for each spectral line. The resulting power spectra are plotted in Fig. 5. These plots indicate the presence of power peaks at around 2,5 and $12 \mathrm{mHz}$ with the largest power peak at around $2 \mathrm{mHz}$. The power decreases at higher frequencies. The peaks could be explained by the fact that any perturbation of the system is represented as a superposition of its normal modes, i.e., 
standing acoustic waves. The periods of these waves are approximately given by the expression

$P=\frac{2 L}{n c_{\mathrm{s}}}, \quad n=1,2, \ldots$

The frequencies of the standing waves are modified by the effects of stratification, thermal and radiative losses, and by the changing sound speed $c_{\mathrm{s}}$ inside the loop. The average temperature inside the loop is about 1.9 MK. Using Eq. (16) we find that the approximate period of the fundamental mode $(n=1)$ is $421 \mathrm{~s}$. This corresponds to a frequency of $2.3 \mathrm{mHz}$. Therefore, the highest peak seen in Figure 5 corresponds to the fundamental mode. The smaller peaks represent the higher harmonics. A more detailed examination of the nanoflare triggered oscillations in different loops will be presented in a forthcoming letter.

These results suggest that the nanoflares may contribute to the power in the observed ranges. However, alternative mechanisms should not be discarded. In particular, a possible source for the $3 \mathrm{mHz}(5 \mathrm{~min})$ oscillations observed in the transition region and corona could be the solar $p$-modes propagating upward along the magnetic flux tubes. Recent studies by De Pontieu et al. $(2004,2005)$ have shown that the photospheric oscillations can actually tunnel through the acoustic cutoff barrier and propagate into the corona so long as they are guided along an inclined magnetic flux tube.

\section{Conclusions}

The present work deals with the nanoflare heating mechanism when the hydrodynamic response of a single loop is investigated. The nanoflares are represented in the form of episodic heating events randomly deposited along the loop. A $40 \mathrm{Mm}$ long loop is considered in which the effects of gravitational stratification, optically thin radiative losses and thermal conduction are taken into account. The loop is initially kept at a constant chromospheric temperature of $20000 \mathrm{~K}$. The initial stage of the loop evolution is completed about $20 \mathrm{~min}$ after the start of the nanoflare heating simulations. During this stage the temperature gradually increases along the loop and the loop is subsequently maintained at coronal temperatures. A narrow and continuously moving transition region is formed. The displacements could be as large as $1 \mathrm{Mm}$. The results are converted into observable quantities by synthesising the emission line profiles for $\mathrm{CIV}$, O VI, Ne VIII and $\mathrm{MgX}$. These resonance lines have peak formation temperatures covering the upper transition region and lower corona. The study reveals that the nanoflare heating could account for the observed dynamic behaviour of the lines. The nanoflare heating could also be responsible for the average red-shifts seen in the transition region. We suggest that the discrepancy between the observed and predicted values for the C IV average Doppler shift could be explained by the fact that the present study does not take into account the non-equilibrium ionisation and footpoint heating. The footpoint heating could also lead to average blue-shifts at higher temperature lines. These effects should be studied in future work. It is shown that the nanoflare heating may contribute to the oscillation power in the observed $\mathrm{mHz}$ ranges. However, the source of the most commonly seen $3 \mathrm{mHz}$ oscillations is probably different. A possible candidate could be the solar $p$ modes propagating upward along the magnetic flux tubes.

Acknowledgements. Research at the Armagh Observatory is grantaided by the N. Ireland Dept. of Culture, Arts and Leisure. YT would like to thank Prof. R. Erdélyi for the initial discussions. YT is also grateful to PPARC for grant PPA/G/S/2002/00020. SJB acknowledges support from a PPARC Rolling Grant. The simulations were run on the Armagh cluster, funded by a PRTLI research grant for Gridenabled Computational Physics of Natural Phenomena (Cosmogrid). CHIANTI is a collaborative project involving the NRL (USA), RAL (UK), and the Universities of Florence (Italy) and Cambridge (UK). The authors thank the anonymous referee for the constructive remarks.

\section{References}

Antiochos, S. K., MacNeice, P. J., Spicer, D. S., \& Klimchuk, J. A. 1999, ApJ, 512, 985

Aschwanden, M. J., \& Parnell, C. E. 2002, ApJ, 572, 1048

Aschwanden, M. J., Schrijver, C. J., \& Alexander, D. 2001, ApJ, 550, 1036

Athay, R. G., \& White, O. R. 1979, ApJ, 229, 1147

Athay, R. G., \& Holzer, T. E. 1982, ApJ, 255, 743

Athay, R. G., \& Dere, K. P. 1989, ApJ, 346, 514

Banerjee, D., O'Shea, E., Doyle, J. G., \& Goossens, M. 2001, A\&A, 371,1137

Bingert, S., Peter, H., Gudiksen, B, et al. 2004, in Proc. SOHO, 15, ESA SP-575, 348

Bradshaw, S. J., \& Mason, H. E. 2003a, A\&A, 407, 1127

Bradshaw, S. J., \& Mason, H. E. 2003b, A\&A, 407, 1127

Bradshaw, S. J., Mason, H. E., \& Del Zanna, G. 2004, A\&A, 425, 287

Brekke, P., Hassler, D. M., \& Wilhelm, K. 1997, Sol. Phys., 175, 349

Cargill, P. J. 1993, Sol. Phys., 147, 263

Cargill, P. J. 1994, ApJ, 422, 381

Cargill, P. J., \& Klimchuk, J. A. 1997, ApJ, 478, 799

Cargill, P. J., \& Klimchuk, J. A. 2004, ApJ, 221, 135

Chae, J., Yun, H. S., \& Poland, A. I. 1998, ApJS, 114, 151

De Pontieu, B., Erdélyi, R., \& James, S. P. 2004, Nature, 430, 546

De Pontieu, B., Erdélyi, R., \& De Moortel, I. 2005, ApJ, 624, L61

Dere, K. P., Bartoe, J.-D. F., \& Brueckner, G. E. 1984, ApJ, 281, 870

Doschek, G. A., Bohlin, J. D., \& Feldman, U. 1976, ApJ, 205, L177

Doyle, J. G., van den Oord, G. H. J., O'Shea, E., \& Banerjee, D. 1998, Sol. Phys., 181, 51

Doyle, J. G., Madjarska, M. S., Roussev, I., Teriaca, L., \& Giannikakis, J. 2002, A\&A, 396, 255

Doyle, J. G., Summers, H. P., \& Bryans, P. 2005a, A\&A, 430, L29

Doyle, J. G., Ishak, B., Ugarte-Urra, I., Bryans, P., \& Summers, H. P. 2005b, A\&A, 439, 1183

Feldman, U., Cohen, L., \& Doschek, G. A. 1982, ApJ, 255, 325

Gudiksen, B. V., \& Nordlund, ̊.. 2005, ApJ, 618, 1020

Hansteen, V. H. 1993, ApJ, 402, 741

Hudson, A. N. 1991, Sol. Phys., 133, 357

Innes, D. E., \& Tóth, G. 1999, Sol. Phys., 185, 127

Judge, P., Carlsson, M., \& Wilhelm, K. 1997, ApJ, 490, L195

Klimchuk, J. A. 1987, ApJ, 323, 368

Klimchuk, J. A., \& Cargill, P. J. 2001, ApJ, 553, 440

Klimchuk, J. A., Antiochos, S. K., \& Mariska, J. T. 1987, ApJ, 320, 409

Mariska, J. T. 1987, ApJ, 319, 465

Mariska, J. T. 1988, ApJ, 334, 489

Mariska, J. T., Feldman, U., \& Doschek, G. A. 1978, ApJ, 226, 698

McClymont, A. N., \& Craig, I. J. D. 1987, ApJ, 312, 402 
Mendoza-Briceño, C. A., Erdélyi, R., Sigalotti, L., \& Di, G. 2002, Sarro, L. M., Erdélyi, R., Doyle, J. G., \& Pérez, M. E. 1999, A\&A, ApJ, 579, L49 351,721

Mendoza-Briceño, C. A., Sigalotti, L., Di, G., \& Erdélyi, R. 2005, ApJ, 624, 1080

Spadaro, D., Noci, G., Zappalà, R., \& Antiochos, S. K. 1990, ApJ, 355,342

Muller, D. A. N., Peter, H., \& Hansteen, V. H. 2004, A\&A, 289, 300

Parker, E. N. 1988, ApJ, 330, 380

Peter, H., \& Judge, P. G. 1999, ApJ, 522, 1148

Peter, H., Gudiksen, B. V., \& Nordlund, Å. 2004, ApJ, 617, L85

Pneuman, G. W., \& Kopp, R. A. 1978, Sol. Phys., 57, 49

Priest, E. R., Foley, C. R., Heyvaerts, J., et al. 1998, Nature, 393, 545

Rosner, R., Tucker, W. H., \& Vaiana, G. S. 1978, ApJ, 220, 643

Spadaro, D., Lanza, A. F., Lanzafame, A. C., et al. 2003, ApJ, 582, 486

Teriaca, L., Banerjee, D., \& Doyle, J. G. 1999a, A\&A, 349, 636

Teriaca, L., Doyle, J. G., Erdélyi, R., \& Sarro, L. M. 1999b, A\&A, 352, L99

Ugarte-Urra, I., Doyle, J. G., Walsh, R. W., \& Madjarska, M. S. 2005, A\&A, 439, 351

Rottman, G. J., Hassler, D. M., Jones, M. D., \& Orrall, F. 1990, ApJ, 358, 69

Walsh, R. W., Bell, G. E., \& Hood, A. W. 1995, Sol. Phys., 161, 83

Walsh, R. W., Bell, G. E., \& Hood, A. W. 1997, Sol. Phys., 171, 81

Roussev, I., Galsgaard, K., Erdélyi, R., \& Doyle, J. G. 2001a, A\&A, 370,298

Withbroe, G. L. 1981, in Activity and Outer Atmospheres of the Sun and Stars, ed. A. O. Benz et al. , 1

Roussev, I., Doyle, J. G., Galsgaard, K., \& Erdélyi, R. 2001b, A\&A, Young, P. R., Del-Zanna, G., Landi, E., et al. 2003, A\&AS, 144, 135 380,719 